\title{
Servicios de indexación y resumen, una oportunidad subvalorada para la mejora de la visibilidad y difusión de los artículos publicados en las revistas científicas Latinoamericanas: caso DOAJ
}

\author{
Juan S. González-Sanabria1, Alicia Aparicio², Diego A. Burgos-Pineda ${ }^{3}$ y Fabián D. Guiza-Pinzón ${ }^{4}$ \\ (1) Facultad de Ingeniería, Escuela de Sistemas y Computación, Universidad Pedagógica y Tecnológica de Colombia, \\ Av. Central del Norte No. 39-115, Tunja, Boyacá, Colombia (correo-e: juansebastian.gonzalez@uptc.edu.co) \\ (2) Consejo Nacional de Investigaciones Científicas y Técnicas (CONICET), Centro Argentino de Información Científica \\ y Tecnológica (CAICYT), Godoy Cruz 2290, Buenos Aires, Argentina (correo-e: aaparicio@conicet.gov.ar) \\ (3) Facultad de Ciencias, Escuela de Física, Universidad Pedagógica y Tecnológica de Colombia, Av. Central del Norte \\ No. 39-115, Tunja, Boyacá, Colombia (correo-e: diegoandres.burgos@uptc.edu.co) \\ (4) Facultad de Ciencias Humanas y Educativas, Departamento de Idiomas, Universidad de Boyacá, Cra. 2 Este No. 64- \\ 169, Tunja, Boyacá, Colombia (correo-e: fdguiza@uniboyaca.edu.co)
}

Recibido Abr. 20, 2020; Aceptado Jun. 15, 2020; Versión final Jul. 13, 2020, Publicado Oct. 2020

\begin{abstract}
Resumen
El objetivo de la presente investigación se centró en analizar el conjunto de datos provistos por el Directorio de Revistas de Acceso Abierto (DOAJ, por sus siglas en ingles), respecto a criterios transversales a la gestión editorial, de cualquier revista independiente de su enfoque, como lo son: cumplimiento de periodicidad, tiempos de respuesta, formatos disponibles, idiomas de publicación, costos de publicación y sometimiento, y manejo de licencias de reproducción. Se realizó un análisis de datos cuantitativo de las 2643 revistas latinoamericanas incluidas en DOAJ a noviembre de 2019. Al analizar los datos de las revistas incluidas en este sistema, se encontró desactualización o falta de precisión en los datos reportados que puede perjudicar la reputación de las revistas. Se concluye, entre otros aspectos, que hay evidencias de demoras en los procesos de revisión y ausencia de formatos interoperables que garanticen el adecuado posicionamiento de los artículos a nivel de la web.
\end{abstract}

Palabras clave: difusión científica; DOAJ; procesos editoriales; revistas científicas; servicios de indexación; visibilidad científica

\section{Indexing and summary services, an undervalued opportunity to improve the visibility and dissemination of articles published in Latin American scientific journals: DOAJ case}

\begin{abstract}
The objective of the present research study focused on analyzing transversal criteria data for editorial management of scientific journals, regardless of their field, that is provided by the Directory of Open Access Journals (DOAJ). The criteria examined included: response time, formats available, publishing language, publication and reviewing costs, and licensing management. A quantitative analysis was performed analyzing the data of the DOAJ journals resulting in 2643 Latin American journals retrieved. Data analyzes showed that there was outdated information or lack of precision at DOAJ that can harm the reputation of journals. It is concluded, among other aspects, that there was evidence of delays in the review processes and that there was an absence of interoperable formats that are required to ensure the proper positioning of articles on the web.
\end{abstract}

Keywords: DOAJ; editorial processes; indexing services; scientific dissemination; scientific journals; scientific visibility 


\section{INTRODUCCIÓN}

Desde la aparición de las publicaciones científicas uno de sus mayores desafíos ha girado en torno a cómo hacer que tengan una mayor visibilidad e impacto, reto que se ha ido facilitando en un mundo que se vuelve cada vez más hacia un contexto digital, puesto que se puede realizar un mejor seguimiento de citaciones, lecturas, temas de interés, entre otros. A nivel mundial son muchas las alternativas que se ofrecen para incluir las revistas en directorios, índices, catálogos y bases de datos, con el fin de dar mayor visibilidad y mejorar la recuperación de la información de los artículos publicados en revistas científicas (González et al, 2019). Algunas de estas iniciativas incluyen las revistas solo con diligenciar un formulario o registrar la revista en el sitio del sistema, y otros requieren de un trámite más complejo que implica que la revista cumpla con una serie de características de calidad en la gestión de sus procesos editoriales y de los contenidos publicados.

En el contexto internacional existen diversos Sistemas de Indexación y Resumen - SIR -, sin embargo, algunos de los de mayor reconocimiento y de alcance multidisciplinar son: Dialnet, DOAJ, Fuente Académica, Latindex, Scopus, Redalyc, REDIB, SciELO y Web of Science (WoS) (Pavas, 2017), los cuales, ya sea de manera automática mediante cosecha de metadatos o manual a través de la carga de la información por parte del equipo de la revista en el portal del sistema, coadyuvan a visibilizar los trabajos publicados en las revistas y, por lo tanto, resultan de bastante utilidad para la difusión internacional de sus contenidos. Sin embargo, también se destaca que existen expertos en cienciometría, como Aguillo (2018), que critican estos sistemas dado que, algunos de estos SIR, en lugar de enlazar el sitio de las revistas incluyen sus contenidos completos (es decir, el texto completo de los artículos), generando que se pueda perder la identidad de cada publicación e incluso afectar las estadísticas de visualización y descarga de los documentos en el sitio web propio.

Es importante resaltar que los SIR de la región han cobrado relevancia y son reconocidos a nivel internacional (Björk, 2017), sin embargo, se debe considerar que cada uno tiene criterios o requisitos independientes para la admisión de una revista a su sistema, por ejemplo, mientras que algunos sistemas como Dialnet, Latindex o REDIB, solo revisan elementos de forma (instrucciones para autores, conformación del comité editorial, tipo de sistema de evaluación, definición de políticas éticas, etc.), otros como Redalyc o SciELO, adicional a los elementos de forma, incluyen en los criterios de inclusión aspectos que van desde la indexación en otras bases de datos y directorios, hasta la revisión de indicadores de endogamia de autores y la calidad de los artículos publicados. Son tan diversos los criterios de admisión de cada SIR que incluso SciELO tiene diferentes cada uno de los países en los que tiene presencia.

Ahora bien, la contribución a la visibilidad que otorgan los SIR va de la mano de un correcto trabajo de los equipos editoriales, pues si bien en estos sistemas encontramos incluidos un sinnúmero de publicaciones, la calidad de la información que se les retorna a los usuarios que hacen uso de estos será directamente proporcional a que tan completa se encuentre incluida la información. Por lo anterior, es necesario analizar las revistas incluidas en los SIR para identificar aquellas que no cumplen con un proceso consecuente y profesional en la carga de información. Como caso de estudio se escogió el Directorio de Revistas de Acceso Abierto DOAJ - por sus siglas en ingles de Directory of Open Access Journals - cuya misión, tal como se declara en su portal, es "aumentar la visibilidad, accesibilidad, reputación, uso e impacto de las revistas revisadas por expertos y de acceso abierto a nivel mundial, independientemente de la disciplina, la geografía o el idioma". DOAJ incluye criterios de validación de las mejores prácticas de publicación y estándares editoriales, manteniendo sus metadatos libres de uso para toda la comunidad. La elección de este Directorio se hizo principalmente por la disponibilidad abierta de los datos de las revistas que incluye (https://www.doaj.org), sumado a que es un Sistema de Indexación y Resumen multidisciplinar y tiene el mayor número de revistas latinoamericanas incluidas que otros sistemas de común uso en la región (Fernández y Murillo, 2018).

A la fecha, se han realizado múltiples estudios relacionados a la presencia de las revistas Latinoamericanas en los SIR, la mayoría se centra ya sea en el análisis de los cuartiles y factor de impacto generado por WoS y Scopus (Vélez-Cuartas et al, 2016) o en la evaluación del comportamiento de un área disciplinar particular, una Institución, o una comunidad (Collazos-Reyes et al, 2017; Ainsworth y Russell, 2018), sin profundizar en el contexto y las características de las publicaciones, como área de actuación, disponibilidad de formatos, cumplimiento de periodicidad y tiempos de respuesta, entre otros, que son aspectos que repercuten en que las revistas tengan una mejor visibilidad y tengan mayor probabilidad de ser consultados y consecuentemente obtener citas de los trabajos que publican, situación que no es posible si los artículos no son recuperables por buscadores, no se pueden leer fácilmente (usabilidad y uso de formatos interoperables) o no cuentan con metadatos disponibles, situación que ocurre en gran parte de Latinoamérica (Salminen et al, 2014; Larivière et al, 2015).

El objetivo de la presente investigación se centró en analizar el conjunto de datos provistos por DOAJ respecto a criterios transversales a la gestión editorial, de cualquier revista independiente de su enfoque, como lo son: 
cumplimiento de periodicidad, tiempos de respuesta, formatos disponibles, idiomas de publicación, costos de publicación y sometimiento, anejo de licencias de reproducción. Dicho análisis es generado con el fin de que los cuerpos editoriales cuenten con elementos que les permitan revisar y reflexionar sobre la importancia de los SIR en sus procesos, la cual va más allá de simplemente estar incluidos, y puedan identificar las falencias comunes que se presentan coadyuvando en mejorar el ciclo de vida de su publicación y de la visibilidad de estas.

\section{METODOLOGÍA}

Se realizó un análisis de datos cuantitativo de las 2643 revistas latinoamericanas incluidas en DOAJ a noviembre de 2019, en la Tabla 1 se puede observar la distribución por países, en donde se puede observar que Brasil es el país que tiene la mayor parte de la producción de revistas registradas en este SIR (53.8\%), y por ende de publicación de artículos en la región (66.5\%). El análisis de datos permite obtener información de las características de las revistas latinoamericanas y realizar una clasificación de los datos disponibles, los cuales, pese a que en gran parte se encuentran estandarizados, fue necesario aplicar un proceso de extracción de la información mediante el uso de expresiones regulares en algunas columnas cuyos datos no se encontraban normalizados.

Los principales criterios con los que cuenta el archivo provisto por DOAJ son: título de la revista; ISSN; eISSN; editorial; áreas temáticas que cubre; número de artículos publicados en el último año; cargos a los autores por envío o procesamiento; política de preservación digital a largo plazo; exploración automática en la revista por buscadores externos; uso de identificadores persistentes a nivel de artículo; disponibilidad de estadísticas a nivel de artículo; formatos de visualización de los textos; información sobre el equipo editorial; sistema de arbitraje; política antiplagio; número de semanas promedio entre el envío y la publicación de los artículos; declaración de adhesión al acceso abierto; licencia de reproducción del texto; declaración de política de derechos de autor; $y$, declaración de los derechos que la revista permite a los autores luego de la publicación del trabajo. Se realizó asimismo una validación aleatoria de los datos registrados en el archivo para detectar posibles errores que se presenten en los mismos, principalmente por falta de actualización.

Tabla 1: Revistas y artículos de revistas latinoamericanas incluidas en DOAJ.

\begin{tabular}{|l|l|l|}
\hline País & Revistas & Artículos \\
\hline Argentina & 240 & 31014 \\
\hline Bolivia & 5 & 326 \\
\hline Brasil & 1423 & 553503 \\
\hline Chile & 120 & 19496 \\
\hline Colombia & 357 & 110862 \\
\hline Costa Rica & 60 & 14820 \\
\hline |Cuba & 91 & 31272 \\
\hline Ecuador & 55 & 9243 \\
\hline El Salvador & 3 & 407 \\
\hline Guatemala & 2 & 235 \\
\hline México & 132 & 36037 \\
\hline Nicaragua & 8 & 1024 \\
\hline Paraguay & 17 & 596 \\
\hline Perú & 65 & 16118 \\
\hline Puerto Rico & 4 & 293 \\
\hline República Dominicana & 5 & 610 \\
\hline Uruguay & 24 & 2553 \\
\hline Venezuela & 32 & 4500 \\
\hline Total & 2643 & 832909 \\
\hline
\end{tabular}




\section{RESULTADOS}

En el presente apartado se realiza el análisis de los datos obtenidos a nivel de la información disponible de DOAJ.

\section{Actualización de metadatos}

El primer análisis se orientó a revisar el nivel de actualización de los artículos publicados en cada revista por país, dicha actualización corresponde al envío automático o manual de los metadatos de cada uno de los artículos para su adecuada recuperación en búsquedas (Castro et al, 2015). Allí se observó que en promedio el $57 \%$ de las revistas dejaron de actualizar su información por un periodo de un año o mayor, incluso, el $29 \%$ de las revistas eligieron no incluir información de los artículos publicados desde su inclusión en DOAJ, como se puede observar en la Tabla 2. Si bien en DOAJ no es un requisito excluyente que las revistas remitan por alguno de los métodos previstos los metadatos a nivel de artículo, sí es deseable por el incremento en la visibilidad y, por consiguiente, en la repercusión de la revista. De los datos se desprende que no parece haber una clara decisión por mejorar la visibilidad de las revistas ya que no se comprometen con la inclusión de los metadatos de los artículos.

Tabla 2: Estado de actualización de las revistas latinoamericanas en DOAJ.

\begin{tabular}{|l|l|l|l|}
\hline País & Sin Actualizar & Más de 1 año & Menos de un año \\
\hline Argentina & $33 \%$ & $35 \%$ & $32 \%$ \\
\hline Bolivia & $20 \%$ & $60 \%$ & $20 \%$ \\
\hline Brasil & $31 \%$ & $26 \%$ & $43 \%$ \\
\hline Chile & $32 \%$ & $38 \%$ & $30 \%$ \\
\hline Colombia & $15 \%$ & $27 \%$ & $58 \%$ \\
\hline Costa Rica & $33 \%$ & $28 \%$ & $38 \%$ \\
\hline Cuba & $32 \%$ & $25 \%$ & $43 \%$ \\
\hline Ecuador & $25 \%$ & $22 \%$ & $53 \%$ \\
\hline El Salvador & $33 \%$ & $67 \%$ & $0 \%$ \\
\hline Guatemala & $0 \%$ & $100 \%$ & $0 \%$ \\
\hline México & $25 \%$ & $34 \%$ & $41 \%$ \\
\hline Nicaragua & $38 \%$ & $25 \%$ & $38 \%$ \\
\hline Paraguay & $59 \%$ & $6 \%$ & $35 \%$ \\
\hline Perú & $23 \%$ & $18 \%$ & $58 \%$ \\
\hline Puerto Rico & $50 \%$ & $50 \%$ & $0 \%$ \\
\hline República Dominicana & $60 \%$ & $20 \%$ & $20 \%$ \\
\hline Uruguay & $42 \%$ & $13 \%$ & $46 \%$ \\
\hline Venezuela & $22 \%$ & $31 \%$ & $47 \%$ \\
\hline Promedio Latinoamérica & $29 \%$ & $28 \%$ & $44 \%$ \\
\hline
\end{tabular}

\section{Área temática}

Este ítem permite conocer la representación y peso de las diferentes áreas temáticas de las revistas científicas latinoamericanas en DOAJ. Para este caso en la Tabla 3 se presenta la primera área reportada por las revistas, lo anterior teniendo en cuenta que en algunas revistas se reportan varias sub-áreas que corresponden a un área de conocimiento definida.

Tabla 3: Distribución por área principal de cobertura temática de las revistas latinoamericanas incluidas en DOAJ.

\begin{tabular}{|l|l|}
\hline Área & Cantidad \\
\hline Agricultura & 155 \\
\hline Educación & 320 \\
\hline Artes & 88 \\
\hline Geografía & 180 \\
\hline Historia & 114 \\
\hline Lenguaje y Literatura & 263 \\
\hline Leyes & 159 \\
\hline Medicina & 301 \\
\hline Filosofía & 152 \\
\hline Ciencias Políticas & 84 \\
\hline Ciencias & 152 \\
\hline Ciencias Sociales & 485 \\
\hline Tecnología & 190 \\
\hline
\end{tabular}


De los resultados presentados se puede observar que la concentración de las publicaciones en las ciencias humanas y sociales alcanza el $69.8 \%$ de las publicaciones en Latinoamérica. El resto se divide entre las ciencias puras con un $5.8 \%$, la ingeniería con $7.2 \%$, medicina $11.4 \%$ y agricultura con $5.8 \%$ de las revistas de la región. Es de resaltar que, si bien algunas revistas reportan múltiples áreas, al hacer la validación de estas de forma "manual", se ubicaron en el área con mayor número de trabajos publicados en el último año.

\section{Cargos a autores por procesamiento}

En Latinoamérica es mayoritario el modelo de acceso abierto en donde es gratuito para los lectores y autores, sin embargo, para la inclusión en DOAJ es necesario informar sobre los valores que cobran la revista o manifestar explícitamente que la revista no genera ningún cargo a los autores. En la Tabla 4 se observa el número de revistas que tienen costo por sometimiento y/o por publicación es de aproximadamente al 3\%, lo cual evidencia el auge y predominio del acceso abierto en Latinoamérica.

Tabla 4: Número de revistas con costo de sometimiento y/o de publicación.

\begin{tabular}{|l|l|l|}
\hline & Costo por sometimiento & Costo por publicación \\
\hline No & 2603 & 2517 \\
\hline $\mathrm{Si}$ & 40 & 126 \\
\hline Total & 2643 & 2643 \\
\hline
\end{tabular}

Sin embargo, para mantenerse en acceso abierto, las revistas han tenido que incurrir en la inclusión de costos de publicación y/o sometimiento que ayuden al financiamiento de estas, dado el limitado apoyo que se tiene por parte de las instituciones editoras y gubernamentales para las revistas científicas (Solomon y Björk, 2016; Pinfield et al, 2015). Para el caso de las revistas objeto del presente análisis, los costos de las revistas que manejan cargos a autores en sus procesos van desde 15 hasta 1.400 U\$S por publicación.

\section{Política de preservación digital}

Debe diferenciarse la preservación digital de las copias de respaldo, donde el segundo término hace referencia a la existencia de una réplica de los datos de un sistema para que el mismo pueda restaurarse en caso de fallas, es decir, funciona como un plan de contingencia (Keller, 2017). En cambio, la preservación digital consiste en una serie de actividades necesarias para asegurar el acceso continuo a los materiales digitales, por el periodo que sea necesario, tanto en caso de fallas como ante cambios tecnológicos, hardware y software. Si bien, para el caso de DOAJ, este no es un requisito de obligatorio cumplimiento por parte de las revistas para su inclusión, si lo es para obtener el sello de calidad DOAJ. En este sentido, se aclara que este Directorio no tiene en cuenta como validos los archivos almacenados en repositorios institucionales y en sistemas en línea propios de la editorial, principalmente, porque estos pueden ser suspendidos en cualquier momento a diferencia de los servicios internacionales como Portico, PKP-PN, LOCKSS, entre otros.

En este sentido, la norma ISO 14721:2012 (ISO, 2012) establece las pautas sobre preservación digital la cual aplican los diferentes sistemas que existen en el mercado, de los cuales pueden distinguirse dos modelos: centralizado (ej. Portico - https://www.portico.org) y descentralizado o distribuido (ej. Cariniana (Silva et al., 2019)). En cuanto a modelos de colaboración externa hay dos opciones: i) subcontratada, servicios de repositorios seguros y por sistemas gestores de preservación digital externos a las organizaciones, y, ii) de asociación, en donde varias organizaciones con los mismos objetivos se comprometen a participar activamente en el desarrollo de servicios compartidos. Así, teniendo en cuenta que los respaldos realizados por las instituciones responsables de las revistas o por sus editores no pueden considerarse preservación, la información declarada en DOAJ por las revistas latinoamericanas es desalentadora, pues 2323 de las 2643 revistas incluidas reporta no tener política de preservación digital. PKP-PN (Sprout y Jordan, 2018) es el sistema de preservación usado por la mayoría de las revistas que manifiestan tener uno (307), se asume que por lo que es el sistema provisto por OJS, (Open Journal System) y tan solo 13 revistas utilizan otro sistema gratuito o de pago.

\section{Exploración automática en la revista por buscadores externos}

Los buscadores como Google Académico y Microsoft Academic (Ortega y Aguillo, 2014), así como los SIR en general necesitan que las revistas permitan la cosecha (harvesting) automática de todos los metadatos de la revista, es decir, la información sobre sí misma, así como las tablas de contenido con títulos de los artículos, resúmenes y palabras clave e identificación de los autores. Si la revista es gestionada y publicada con OJS bastará habilitar esta opción que ya prevé el software. En este criterio es donde se presenta la mayor inconsistencia y falta de seguimiento por parte de DOAJ, puesto que pese a que 2004 de las revistas aseguran disponer de mecanismos de exploración automática, los datos de los artículos de cada revista tienen serios 
problemas de actualización, lo cual lleva a deducir que DOAJ no hace la captura de metadatos adecuadamente o los equipos editoriales no configuran adecuadamente la cosecha de datos, haciendo que los datos no se actualicen permanentemente.

Es de aclarar que DOAJ no exige el uso de software/araña para el rastreo de contenido, sin embargo, si una revista es aceptada y no los usa, debe comprometerse a enviar los metadatos de todos sus artículos a DOAJ en los 3 siguientes meses a la aceptación. En este sentido, más que evaluar si el Directorio pide o no el uso de software que permitan la minería de datos, las revistas deben hacer uso de estos, pues permiten la fácil recuperación e indexación en buscadores de manera automática, caso contrario la revista difícilmente se recuperará por parte de los investigadores y lectores en la exploración que hagan en la Web para sus proyectos.

\section{Identificador persistente a nivel de artículo}

Ante la gran volatilidad en el ámbito web es necesario asegurar de manera permanente la localización y acceso a los recursos u objetos digitales, independiente del cambio de ubicación que, a lo largo del tiempo, pueda experimentar un artículo o documento. En relación con este parámetro DOAJ prevé principalmente tres opciones de identificadores: DOI, Handle y ARK. De los tres mencionados el único que no requiere de un servicio externo para su configuración o uso, y por lo tanto es gratuito, es el ARK, no obstante, este no es utilizado en Latinoamérica como puede verse en la Tabla 5. Se observa un gran número de revistas que no usa ningún tipo de identificador, un hecho preocupante para la recuperación de los artículos, y más aún cuando se habla de revistas que reportan ser electrónicas.

Tabla 5: Revistas con uso de identificador permanente de artículo.

\begin{tabular}{|l|l|}
\hline Identificador & Cantidad \\
\hline DOI & 937 \\
\hline Handle & 10 \\
\hline ARK & 0 \\
\hline Ninguno & 1696 \\
\hline
\end{tabular}

\section{Formatos de salida de los textos completos}

Pese a que en los últimos años se viene hablando de una migración de publicación impresa a electrónica en Latinoamérica para contribuir con la visibilidad y difusión de los contenidos, sin embargo, al revisar los formatos electrónicos como PDF, XML y HTML (Tabla 6) se observa que la mayoría de las revistas asocian el formato digital con disponer de los artículos en formato PDF, cuando incluso este formato de archivo no tiene metadatos asociados o son de difícil lectura. Al hablar de formatos especializados como XML, el número de revistas que trabajan en este es de tan solo el $11.4 \%$, lo que puede repercutir en la dificultad de indexación por parte de los robots o spiders con los que trabajan los buscadores como Google.

Tabla 6: Disponibilidad de formatos electrónicos.

\begin{tabular}{|l|l|l|l|l|}
\hline \multirow{2}{*}{ Formato } & \multicolumn{2}{|l|}{ Revistas que lo usan } & \multicolumn{2}{l|}{ Revistas que no lo usan } \\
\cline { 2 - 5 } & Cantidad & Porcentaje & Cantidad & Porcentaje \\
\hline PDF & 2636 & 99.7 & 7 & 0.3 \\
\hline XML & 302 & 11.4 & 2341 & 88.6 \\
\hline HTML & 697 & 26.4 & 1946 & 73.6 \\
\hline
\end{tabular}

\section{Información sobre el equipo editorial}

Una buena práctica editorial es que el equipo editorial (Consejo Editorial, Comité Editorial, Académico, Científico, etc.) encargado de la gestión de la revista se presente con la información más completa posible. Esto incluye, además de nombres y apellidos, la filiación institucional completa y una dirección de correo electrónico de contacto. Adicionalmente, puede enlazarse a un perfil en una red académica o institucional o a ORCID. En este punto DOAJ requiere que todas las revistas cuenten con un Editor y un Consejo editorial, el cual debe estar conformado por al menos 5 integrantes, todos los miembros deben estar debidamente identificado con su filiación institucional y su correo electrónico, datos que se tienen que mencionar en la Web de la revista, sin embargo, se aclara que el Directorio, para el caso de revistas de artes y humanidades aplica la excepción de que existan dos editores en lugar de un editor y un Consejo editorial. 


\section{Sistema de arbitraje}

La evaluación por pares es la forma de control por excelencia de la calidad científica y de posibles errores que presenten los artículos antes de su publicación (Rozemblum et al., 2015). Es un proceso crítico y dinámico de regulación de la calidad basado en el juicio científico de otros expertos quienes, además, son ellos mismos investigadores en el área temática del trabajo que van a evaluar. El arbitraje busca seleccionar para la publicación los trabajos competentes, significativos y originales (Sense About Science 2014). DOAJ prevé diferentes modalidades de trabajo para la evaluación de los artículos científicos: ciego, doble ciego, abierto, pero también acepta revistas cuya evaluación es realizada por el mismo equipo editorial, algo que no es aceptable por varios de los SIR (por ejemplo, Scopus y SciELO).

Tabla 7: Sistema de arbitraje utilizado en revistas latinoamericanas.

\begin{tabular}{|l|l|}
\hline Tipo de arbitraje & Cantidad \\
\hline Revisión Ciega Simple & 415 \\
\hline Revisión Doble Ciega & 1776 \\
\hline Revisión Editorial & 58 \\
\hline Revisión Abierta & 394 \\
\hline Total & 2643 \\
\hline
\end{tabular}

\section{Política antiplagio}

Uno de los controles que debe realizar la revista cuando recibe la postulación de un artículo para la publicación es chequear que el trabajo no haya sido publicado ya en forma total o en parte o que incurra en alguna práctica reñida con la ética. Es decir, se trata de localizar en un texto dado partes tomadas de otras fuentes 0 documentos, pero sin incluir la cita o referencia correspondiente (Spinak, 2014; Graf et al, 2007). La detección de plagio, gracias a la existencia de software especializado, se facilita cada vez más dada la ingente cantidad de información se almacena actualmente de manera digital, sea en revistas científicas como tesis en repositorios institucionales y otros sitios como blogs y bibliotecas virtuales, por lo que se crearon aplicaciones para facilitar este control. Hay aplicaciones gratuitas, como Plagiarisma (https://plagiarisma.net), Plag.es (https://plag.es) o Viper (https:// https://plag.co/), para descargar y también para utilizar en línea, y también programas por suscripción. DOAJ requiere en este ítem que se explique cuál es la política antiplagio de la revista, incluyendo el medio con el que se realiza la verificación y si se aplica alguna penalidad en caso de detectarse. Para este caso, solo $722(27.3 \%)$ revistas manifiestan tener una política claramente definida para cuando los casos de plagio o autoplagio se detectan.

\section{Número de semanas promedio entre el envío y la publicación de los artículos}

Una dificultad que suelen reportar los autores al momento de enviar sus trabajos a revistas editadas en Latinoamérica se relaciona con los tiempos de respuesta de estas (Tabla 8), puesto que el número de meses en los que una revista responde si el artículo es o no publicado puede tardar en algunos países más de cinco meses, que pueden representar una pérdida de la "innovación" de los resultados presentados en sus artículos. Este aspecto en realidad da una idea de la mayor o menor profesionalización del equipo editorial, de cómo se organiza y cuán bien funciona para cumplir con el ciclo de edición. Es un ítem que los autores toman en consideración al momento de seleccionar una revista objetivo para enviar el resultado de su investigación. Como puede verse en la Tabla 8, el tiempo promedio de respuesta a los autores por parte de las revistas latinoamericanas es de casi 4 meses (3.9), e incluso 5 meses o más en países como Colombia, Guatemala y México. Si bien lo anterior puede considerarse un tiempo normal de espera por algunos investigadores, en áreas de conocimiento como medicina, ingeniería y ciencias puras, un tiempo de espera mayor a dos meses en la publicación de un artículo llega a ser crítico, pues puede que el tema sea novedoso y alguien más lo publique o que el mismo pierda su vigencia.

Tabla 8: Tiempo promedio de respuesta de aceptación o rechazo de un artículo, contado desde el envío.

\begin{tabular}{|l|l|l|}
\hline País & Semanas & Meses \\
\hline Argentina & 17.1 & 4.3 \\
\hline Bolivia & 12.2 & 3.1 \\
\hline Brasil & 18.5 & 4.6 \\
\hline Chile & 16.8 & 4.2 \\
\hline Colombia & 19.5 & 4.9 \\
\hline
\end{tabular}


Tabla 8: continuación.

\begin{tabular}{|l|l|l|}
\hline País & Semanas & Meses \\
\hline Costa Rica & 16.8 & 4.2 \\
\hline Cuba & 13.1 & 3.3 \\
\hline Ecuador & 12.9 & 3.2 \\
\hline El Salvador & 13.7 & 3.4 \\
\hline Guatemala & 21.0 & 5.3 \\
\hline México & 21.4 & 5.4 \\
\hline Nicaragua & 12.6 & 3.2 \\
\hline Paraguay & 12.6 & 3.1 \\
\hline Perú & 13.0 & 3.3 \\
\hline Puerto Rico & 13.8 & 3.4 \\
\hline República Dominicana & 11.2 & 2.8 \\
\hline Uruguay & 16.5 & 4.1 \\
\hline Venezuela & 14.6 & 3.7 \\
\hline Promedio Latinoamérica & 15.4 & 3.9 \\
\hline
\end{tabular}

\section{Licencia de reproducción del contenido}

DOAJ requiere que se declare en la revista qué permisos concede a los lectores, y puede hacerse mediante la adopción de alguna de las seis opciones de licencias Creative Commons (CC) o en su defecto otra, que puede ser propia de la institución responsable de la revista, pero de similares características. De lo anterior se puede evidenciar que la mayoría de las revistas optan por una difusión de los trabajos menos restrictiva como es el uso de la licencia CC BY, lo que se conoce como la opción más abierta.

Tabla 9: Licencia de reproducción usadas por las revistas.

\begin{tabular}{|l|l|}
\hline Licencia & Cantidad \\
\hline CC BY & 1009 \\
\hline CC BY-NC & 640 \\
\hline CC BY-NC-ND & 503 \\
\hline CC BY-NC-SA & 397 \\
\hline CC BY-ND & 18 \\
\hline CC BY-SA & 67 \\
\hline Licencia Propia & 9 \\
\hline
\end{tabular}

\section{DISCUSIÓN}

Por lo presentado a nivel del análisis de cada uno de criterios principales, SIR como DOAJ, que, si bien realiza una labor dedicada y detallada al momento de aceptar una revista para la inclusión, se dejan de lado los procesos después de la inclusión, allí se deberían establecer criterios para permanecer, como por ejemplo tiempos para actualizar la información o de lo contrario retirar estás publicaciones.

Para soportar lo anterior, al profundizar en Colombia, uno de los países en los que se considera a DOAJ como SIR, y que permite dar cumplimiento a requisitos mínimos para ser reconocida una revista científica en dicho país, se encontró que, de las 357 publicaciones incluidas en el Directorio, al realizar una revisión "manual" de cada revista, se encontraron varios errores en la información reportada respecto a la mostrada en el sitio de cada revista. Para iniciar, 27 de ellas mencionan que sus sistemas de gestión editorial es SciELO o Redalyc, sin embargo, al ingresar a los respectivos sitios web, el sistema usado es OJS; más crítico aún es el hecho de que 29 revistas no mencionan ningún sistema de publicación. Un segundo hecho que se observó es que pese a que todas las revistas cuentan con un sitio electrónico 85 revistas reportan tener solo ISSN impreso, lo cual no es coherente con un mundo que se mueve en lo computacional. Este caso se toma como el reflejo de las publicaciones en Latinoamérica, pues en términos de los datos registrados en DOAJ, pues se insiste en que las mismas han migrado a una publicación electrónica, la mayoría apenas cuenta con una versión en PDF de sus artículos y un número mínimo asigna un identificador a sus artículos que ayude a que los mismos se identifiquen de manera unívoca y se puedan recuperar rápidamente en la Web.

Puede ser interesante revisar el por qué los evaluadores de ciencia de cada país no comparan o usan las áreas reportadas en los SIR, como DOAJ, para evaluar la veracidad de la información reportada; lo anterior se plantea dado que al verificar las áreas reportadas por la revistas en DOAJ y las reportadas por las mismas 
en los catálogos nacionales e internacionales se encontró, para el caso de ejemplo de Colombia, que al menos $37 \%$ de las publicaciones reportan áreas diferentes en los dos sistemas, lo que puede llegar a indicar una manipulación de la información para lograr su categorización a nivel del catálogo nacional, puesto que Minciencias no realiza validación de que los contenidos publicados si correspondan con el área reportada, implicando que, al parecer, los editores reporten un área "conveniente" de su revista en su catálogo Publindex para verse favorecido en otros criterios como el de citación.

A nivel de la actualización de datos, parece que la mayoría de las revistas solo se interesan por incluir las revistas en los SIR, mas no porque los datos se actualicen continuamente, en el caso de estudio de DOAJ, más del $50 \%$ de las publicaciones han dejado de incluir su información desde hace más de un año o nunca la ha actualizado, lo que hace perder visibilidad y credibilidad en la continuidad de la publicación. Este detalle es de particular atención cuando se revisa, que el $70 \%$ de las revistas incluidas en el Directorio en el periodo 2017-2019, nunca han incluido los metadatos de sus artículos, evidenciando una falta de compromiso de los editores, quienes no adquieren el compromiso de actualizar la información, es decir, usan DOAJ para el cumplimiento de unos requisitos de medición de índices nacionales u otros SIR, pero no para dar una adecuada visibilidad de la publicación, desaprovechando las bondades de difusión que el mismo ofrece. En el caso de las revistas en la plataforma SciELO, algunos de los sitios SciELO certificados, como SciELO Argentina, requieren que, para su incorporación y mantenimiento, las revistas estén previamente indexadas en DOAJ. Lo anterior, ha ocasionado que se incrementen las solicitudes para la inclusión de las revistas latinoamericanas en este Directorio.

Otro aspecto para tener en cuenta es la reciente tendencia a incluir APC por parte de las revistas Latinoamericanas para poder mantenerse en acceso abierto, en gran parte esto se puede ocasionar por la falta de iniciativas conjuntas de edición entre Instituciones, pues según la información reportada por las revistas Latinoamericanas en DOAJ aproximadamente solo un $1.2 \%$ es editado por más de una institución, llevando a que los recursos se dispersen y encontrando que en una sola ubicación geográfica (a nivel de país) se lleguen a encontrar hasta 60 revistas del mismo campo de acción, cada una con 10 artículos al año, en lugar de fusionarse y optimizar recursos. Lo anterior lleva a la necesidad de revisar y unificar criterios en las políticas de producción científica de los países de Latinoamérica, tal como lo afirman Vessuri, Guédon y Cetto (2014) se "requiere prestar especial atención a los mecanismos que sustentan la producción, circulación y consumo de las revistas científicas" en nuestros países con el objetivo de hacer las revistas más competitivas y a adquirir mayor reputación a nivel internacional y no solo a nivel de la región.

En cuanto a la migración de revistas de impreso a electrónica (Wouters et al., 2006), es necesario generar compromiso por parte de los equipos editoriales en el sentido de que la migración va más allá de poner disponible el PDF del artículo, se requiere de formatos que sea interoperables, como XML-JATS, para facilitar la recuperación de la información y la cosecha de metadatos de manera exitosa, garantizando trazabilidad en consultas y citas a largo plazo.

\section{CONCLUSIONES}

De acuerdo con el trabajo presentado y a los resultados obtenidos, se pueden plantear las siguientes conclusiones principales:

1.- Si bien los SIR traen consigo grandes ventajas para la difusión de los artículos en la comunidad internacional al ser ampliamente consultados por académicos e investigadores para fundamentar sus procesos científicos, un elevado porcentaje $(57 \%)$ de las revistas latinoamericanas incluidas en DOAJ no actualizan hace más de un año, o nunca han actualizado, sus contenidos en el Directorio, lo que repercute en que los mismos no aparezcan en los resultados de las búsquedas realizadas por los usuarios del sistema.

2.- Es en este sentido que las instituciones editoras deben realizar una inversión en capacitación o contratación de personal técnico que ayude en la revista en la adecuada configuración de recuperadores automáticos de minería para garantizar la adecuada cosecha de los artículos de la revista, no solo a nivel de los SIR, si no a nivel de los buscadores web en general.

3.- Se requiere una amplia sensibilización en el uso de formatos electrónicos y digitales de los artículos de las revistas que vayan más allá de la publicación de la versión en PDF, pues las revistas a nivel latinoamericano mencionan que son revistas electrónicas o que han migrado a esta versión, en sus sitios no disponen de formatos de mayor recuperabilidad a nivel web como XML.

4.- Se deben generar estrategias, como Marcalyc de Redalyc, que sirven para la generación del contenido en formato XML, el cual es de amplio uso a nivel internacional y se complementa con estándares como JATS (Journal Article Tag Suite), permitiendo describir la estructura y el contenido de los artículos de una revista. 
5.- El tiempo promedio de respuesta de las revistas latinoamericanas incluidas en DOAJ es de aproximadamente 4 meses, en publicaciones con enfoque en los temas de Filosofía, Historia y Geografía oscila entre los 6 y 7 meses. Los tiempos de respuesta desde el envío de un artículo hasta su publicación deben ser revisados e informados por las revistas a los autores, con el fin de que los investigadores cuenten con la información necesaria al momento de elegir en donde publicar.

6.- El uso del sistema de publicación continua (como PLoS ONE), debería evaluarse por los editores de las revistas latinoamericanas para agilizar los tiempos de publicación y no esperar a la consolidación de un numero o volumen para la difusión de los resultados.

7.- Se debe buscar, inicialmente por países, la fusión de revistas que tengan el mismo enfoque y área de cobertura que permita aunar esfuerzos económicos para que, por ejemplo, en lugar de publicar 10 revistas cada una con 10 artículos al año, se publique una revista de mayor envergadura.

8.- Se debe optimizar los recursos e invertirlos en fortalecer las capacidades editoriales y técnicas de la revista en los desafíos de visibilidad y posicionamiento que demandan las publicaciones en una era digital a la que se le debe sacar un máximo provecho para aumentar el reconocimiento y la difusión de estas.

\section{REFERENCIAS}

Aguillo, I., Open Science: Retos y Oportunidades, VI Congreso Internacional de Tecnologías Emergentes y Sociedad, Logroño, España (2018)

Ainsworth, S. y Russell, J., Has hosting on science direct improved the visibility of Latin American scholarly journals? A preliminary analysis of data quality. https://doi.org/10.1007/s11192-018-2725-7, Scientometrics, 115, 1463-1484 (2018)

Björk, B.-C., Journal Portals-An Important Infrastructure for Non-commercial Scholarly Open Access Publishing. https://doi.org/10.1108/OIR-03-2016-0088, Online Information Review, 41(5), 643-654 (2017)

Castro-Romero, A., González-Sanabria, J. S. y Ballesteros-Ricaurte, J. A., Technologies for Metadata Management in Scientific Articles. https://doi.org/10.25100/iyc.v17i2.2195, Ingeniería y Competitividad, 17 (2), 123-134 (2015)

Collazo-Reyes, F., Luna-Morales, M. y Luna-Morales, E., Change in the publishing regime in Latin America: from a local to universal journal Archivos de investigación Médica/Archives of Medical Research (1970-2014). https://doi.org/10.1007/s11192-016-2207-8, Scientometrics, 110, 695-709 (2017)

Fernández, M. y Murillo, D., Análisis Bibliométrico de las Revistas de Acceso Abierto de Centroamérica, el Caribe y México Basado en DOAJ e Indexadores Latinoamericanos, Memorias de Congresos UTP, ISBN: 978-9962-698-57-9, 1(1), 57-64 (2018)

González-Sanabria, J. S., Díaz-Peñuela, J. S., y Castro-Romero, A, Análisis de los Indicadores de Citación de las Revistas Científicas Colombianas en el Área de Ingeniería. https://doi.org/10.4067/S0718-07642019000200293, Información Tecnológica, 30 (2), 293-302 (2019)

Graf, C., Wager, E. y otros cuatro autores, Best Practice Guidelines on Publication Ethics: a Publisher's Perspective. https://doi.org/10.1111/j.1742-1241.2006.01230.x, International Journal of Clinical Practice, 61 (s152), 1-26 (2007)

ISO 14721:2012: International Organization for Standardization (Suiza), Space Data and Information Transfer SystemsOpen Archival Information System (OAIS); 1-25, Ginebra-Suiza (2012)

Keller, T., Review of Digital Preservation Essentials, Journal of Western Archives, ISSN: 2154-7149, 8 (1), 2 (2017)

Larivière, V., Haustein, S. y Mongeon, P., The Oligopoly of Academic Publishers in the Digital Era. https://doi.org/10.1371/journal.pone.0127502, PLoS ONE, 10 (6), e0127502 (2015)

Ortega, J. L. y Aguillo, I. F., Microsoft Academic Search and Google Scholar Citations: Comparative Analysis of Author Profiles. https://doi.org/10.1002/asi.23036, J Assn Inf Sci Tec, 65: 1149-1156 (2014)

Pavas, A., Report About the Preliminary Results of the National Bibliographic Index - Publindex, Ingeniería e Investigación, ISSN: 0120-5609, 37 (1), 1-6 (2017)

Pinfield, S., Salter, J. y Peter A., The "Total Cost of Publication" in a Hybrid Open-Access Environment: Institutional Approaches to Funding Journal Article-Processing Charges in Combination with Subscriptions. https://doi.org/10.1002/asi.23446, Journal of the Association for Information Science and Technology, 67 (7), $1751-1766$ (2016)

Rozemblum, C., Unzurrunzaga, C., Banzato, G. y Pucacco, C., Calidad Editorial y Calidad Científica en los Parámetros para Inclusión de Revistas Científicas en Bases de Datos en Acceso Abierto y Comerciales, Palabra Clave (La Plata), ISSN: 1853-9912, 4(2), 64-80 (2015)

Salminen, A., Jauhiainen, E. y Nurmeksela, R., A life cycle model of XML documents, https://doi.org/10.1002/asi.23148, Journal of the Association for Information Science \& Technology, 65 (12), 2564-2580 (2014) 
Sense About Science, Peer Review and the Acceptance of New Scientific Ideas. Discussion Paper from a Working Party on Equipping the Public with an Understanding of Peer Review, Londres, Inglaterra (2014)

Silva, M. P., De Moura, R. K., Siebra, S. y Pinto, V. B., Contribuições da Rede Cariniana para a Preservação Digital nos Repositórios Digitais Institucionais, Informação em Pauta, ISSN: 2525-3468, 4 (Especial 2), 99-116 (2019)

Solomon, D. y Björk, B.-C., Article Processing Charges for Open Access Publication-The Situation for Research Intensive Universities in the USA and Canada, https://doi.org/10.7717/peerj.2264, PeerJ, 4, e2264 (2016)

Spinak, E., Ética Editorial ¿Cómo Detectar el Plagio por Medios Automatizados?, Brasil (2014)

Sprout, B. y Jordan, M., Distributed Digital Preservation: Preserving Open Journal Systems Content in the PKP PN. https://doi.org/10.1108/DLP-11-2017-0043, Digital Library Perspectives, 34 (4), 246-261 (2018)

Vélez-Cuartas, G., Lucio-Arias, D. y Leydesdorff, L., Regional and global science: Publications from Latin America and the Caribbean in the SciELO Citation Index and the Web of Science. https://doi.org/10.3145/epi.2016.ene.05, El profesional de la información, 25 (1), 35-46 (2016)

Vessuri, H., Guédon, J.-C. y Cetto, A. M., Excellence or Quality? Impact of the Current Competition Regime on Science and Scientific Publishing in Latin America and its Implications for Development, https://doi.org/10.1177/0011392113512839, Current Sociology, 62(5), 647-665 (2014)

Wouters, P., Reddy, C. y Aguillo, I., On the visibility of information on the Web: an exploratory experimental approach. https://doi.org/10.3152/147154406781775931, Research Evaluation, 15(2), 107-115 (2006) 
